\title{
Dynamic electron-phonon and spin-phonon interactions due to inertia
}

\author{
R. Matthias Geilhufe [1] \\ Nordita, KTH Royal Institute of Technology and Stockholm University, Roslagstullsbacken 23, 10691 Stockholm, Sweden
}

(Received 3 August 2021; revised 2 December 2021; accepted 7 December 2021; published 14 January 2022)

\begin{abstract}
$\mathrm{THz}$ radiation allows for the controlled excitation of vibrational modes in molecules and crystals. We show that the circular motion of ions introduces inertial effects on electrons. In analogy to the classical Coriolis and centrifugal forces, these effects are spin-rotation coupling, centrifugal field coupling, centrifugal spin-orbit coupling, and centrifugal redshift. Depending on the phonon decay, these effects persist for various picoseconds after excitation. Potential boosting of the effects would make it a promising platform for vibration-based control of localized quantum states or chemical reaction barriers.
\end{abstract}

DOI: 10.1103/PhysRevResearch.4.L012004

In the adiabatic Born-Oppenheimer approximation, the electronic degrees of freedom are separated from the ionic degrees of freedom. Excitations of the ionic degrees of freedom such as phonons in crystals and vibrations, torsions, and rotations in molecules can be resonantly driven by state-ofthe-art experiments with coherent high intensity $\mathrm{THz}$ laser beams [1,2]. This opens the prospect of coherent control of emergence in quantum materials and the design of new phases of matter [3-5]. For example, the coherent control of local dipoles induce a transient magnetization in the framework of dynamical multiferroicity [6,7]. The strength of this effect is predicted to be in the order of the nuclear magneton [7-9]. However, recent experiments measuring the magneto-optical Kerr effect hint toward much higher magnetic moments [10]. In the phonon Zeeman effect, left- and right-handed circularly polarized phonon modes split in a magnetic field. Again, the measured phonon Zeeman splitting seems to be much larger than theoretical estimates [11-16]. This discrepancy has initiated a debate on phonon angular momenta [17-19], nonlinear or anharmonic corrections [15], topological features of effective charges [20], and angular momentum transfer from ionic to electronic degrees of freedom [9].

Electron-phonon interactions are commonly described using the Fröhlich Hamiltonian [21], which can be extended toward including spin-phonon terms [22]. In the present paper we aim to provide a different microscopic model coupling ionic motion to electronic degrees of freedom. This model is based on inertial effects experienced by electrons bound to a moving ion. Considering the circular motion of an ion due to a phonon excitation (see Fig. 1), a local inertial frame accelerates relative to a selected ion. However, a local observer on the ion finds themselves in a noninertial

Published by the American Physical Society under the terms of the Creative Commons Attribution 4.0 International license. Further distribution of this work must maintain attribution to the author(s) and the published article's title, journal citation, and DOI. frame. As a result, inertial effects emerge. In classical mechanics, a rotating coordinate system induces fictious forces like the Coriolis force $\boldsymbol{F}_{\text {Coriolis }}=2 \boldsymbol{p} \times \boldsymbol{\omega}$ or the centrifugal force $F_{\text {Cent. }}=m \omega^{2} \boldsymbol{d}$, where $\boldsymbol{\omega}(\omega)$ is the angular velocity (frequency), $\boldsymbol{d}$ is the radius determined by the ionic displacement from the equilibrium position, $\boldsymbol{p}$ is the momentum of a probe particle, and $m$ is the corresponding mass. Promoting the fictious forces to an energy by multiplying with the position of the probe particle reveals the coupling of the angular velocity with the probe particle angular momentum $E=\boldsymbol{F}_{\text {Coriolis }} \cdot \boldsymbol{r}=$ $2 \omega \cdot \boldsymbol{L}$ or a centrifugal-force coupling comparable to the coupling of an applied electric field, $E=\boldsymbol{F}_{\text {Cent. }} \cdot \boldsymbol{r}=m \omega^{2} \boldsymbol{d} \cdot \boldsymbol{r}$. Subsequently, we review that the Coriolis and centrifugal effects also emerge in quantum systems and provide realistic estimates for resulting electron-phonon and spin-phonon interactions.

Inertial effects of quantum systems have attracted great attention in astronomical settings or collision experiments. Related to the classical Coriolis force, the rotation of a quantum system leads to the well-known spin-rotation coupling $\sim \boldsymbol{\omega} \cdot \boldsymbol{J}$, with $\boldsymbol{J}=\boldsymbol{L}+\boldsymbol{S}$ the total angular momentum. Variants of this coupling were derived by Werner et al. [26], Mashoon [27], Müller and Greiner [28], Hehl and Ni [29], and Ryder [30]. Spin-rotation coupling has been observed using neutron interferometry $[27,31]$, where two perpendicular neutron beams accumulate a phase shift due to the earth rotation. Note that the rotation frequency of the earth $\approx 70 \mu \mathrm{Hz}$ is about 17 orders of magnitude smaller than the ionic motion in a crystalline lattice. Quantum effects for accelerating references frames were discussed, e.g., by Hehl and $\mathrm{Ni}$ [29], Hehl [32], and Ryder [30]. With the prospect of mechanically inducing a topological phase and spin currents, the idea of tuning quantum states by accelerations has been developed, e.g., for samples coupled to microwave resonators, inducing tiny circular motions [33,34]. Inertial effects of spins were recently discussed in the context of spin dynamics and the Landau-Lifshitz-Gilbert equation, describing, e.g., spin nutations [35-38].

To discuss the coupling of the ionic motion to the electron, we focus on a single ion moving on a circular orbit 

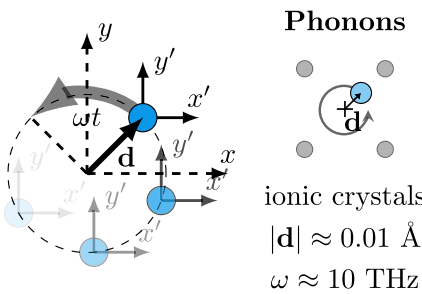

Torsions

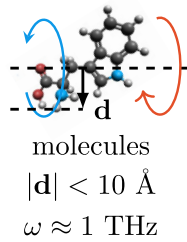

FIG. 1. A circularly polarized laser pulse induces the controlled motion of ions. We show the frequency and the rotation radius for selected examples. Estimates taken in agreement with Refs. [7,9,23-25].

around the Cartesian $z$ axis, with frequency $\omega$ and displacement $\boldsymbol{d}=d(\cos \omega t, \sin \omega t, 0)$. Examples are summarized in Fig. 1. To obtain access to the spin degrees of freedom, we follow the formalism of Hehl and $\mathrm{Ni}$ [29] and formulate the corresponding Dirac equation in an accelerating frame and take the nonreativistic limit to the Schrödinger equation.

By definition of the coordinate system, we obtain for the four-velocity and the four-acceleration

$$
u^{\mu}=\gamma(c, \omega \times \boldsymbol{d}), \quad a^{\mu}=\left(0,-\omega^{2} \gamma^{2} \boldsymbol{d}\right),
$$

with the Lorentz factor $\gamma=\left(1-\frac{d^{2} \omega^{2}}{c^{2}}\right)^{-\frac{1}{2}} \approx 1$. The coordinate tetrad $e_{a}$ carried along with the observer is a rest frame for the observer. Hence the observer's time axis coincides with the four-velocity $e_{0}^{\mu}=u^{\mu}$. At the same time, the tetrad $e_{\mu}$ must remain orthonormal. We apply generalized FermiWalker transport to describe the accelerating and rotating observer and the tetrad transported along the observer's world line, parametrized by the proper time $\tau$,

$$
\frac{\mathrm{d} e_{a}}{\mathrm{~d} \tau}=\Omega_{\mathrm{FW}} \cdot e_{a}
$$

Here the generalzed Fermi-Walker transport tensor $\Omega_{\mathrm{FW}}$ splits into a nonrotating and a rotating part $[39,40]$

$$
\Omega^{\mu \nu}=\frac{1}{c^{2}}\left(a^{\mu} u^{\nu}-a^{\nu} u^{\mu}\right)+u^{\alpha} \omega^{\beta} \epsilon^{\alpha \beta \mu \nu} .
$$

Here $\epsilon^{\alpha \beta \mu \nu}$ is the antisymmetric Levi-Civita tensor. The infinitesimal line element is

$$
\begin{aligned}
d s^{2}= & \left(d x^{0}\right)^{2}\left[\left(1-\omega^{2} \gamma^{2} \boldsymbol{d} \cdot \boldsymbol{r}\right)^{2}-(\boldsymbol{\omega} \times \boldsymbol{r})^{2}\right] \\
& -\frac{2}{c} \boldsymbol{\omega} \times \boldsymbol{r} d x^{0}-d \boldsymbol{r} \cdot d \boldsymbol{r} .
\end{aligned}
$$

In the observer's local frame, the Dirac equation is written as

$$
\gamma^{a} \mathrm{i} \hbar D_{a} \Psi=m c \Psi
$$

Here $\Psi$ is a four-spinor, $m$ is the electron mass, and $\gamma^{a}$ is the Dirac $\gamma$-matrices, $\left\{\gamma^{\mu}, \gamma^{\nu}\right\}=\eta^{\mu \nu} . D_{a}=\partial_{a}-\frac{\mathrm{i}}{4} \sigma^{b c} \Gamma_{b c a}$ is the covariant derivative, with $\sigma^{a b}=\frac{\mathrm{i}}{2}\left[\gamma^{a}, \gamma^{b}\right]$ and $\Gamma_{b c a}$ the connection coefficient [29,32].

Following Refs. [29,39] and evaluating Eq. (5) for our example gives the following Dirac equation including inertial

terms,

$$
\begin{aligned}
\mathrm{i} \hbar \partial_{t} \Psi= & {\left[c \boldsymbol{\alpha} \cdot \boldsymbol{p}-\frac{\gamma^{2}}{2 m c}\left\{\boldsymbol{F}_{\text {Cent. }} \cdot \boldsymbol{r}, \boldsymbol{p} \cdot \boldsymbol{\alpha}\right\}\right.} \\
& \left.+\beta\left[m c^{2}-\gamma^{2}\left(\boldsymbol{F}_{\text {Cent. }} \cdot \boldsymbol{r}\right)\right]-\boldsymbol{\omega} \cdot \boldsymbol{J}\right] \Psi .
\end{aligned}
$$

Note that we now use the Dirac matrices $\alpha_{i}$ and $\beta$, with $\left\{\alpha_{i}, \alpha_{j}\right\}=\left\{\alpha_{i}, \beta\right\}=0$ and $\alpha_{i}^{2}=\beta^{2}=1$. They are related to the $\gamma$ matrices by $\gamma^{0}=\beta$ and $\gamma^{i}=\beta \alpha_{i}$. The conventional Dirac Hamiltonian $H=c \boldsymbol{\alpha} \cdot \boldsymbol{p}+\beta m c^{2}$ is extended by three dynamic terms. The first two dynamic terms correspond to the centrifugal force $\boldsymbol{F}_{\text {Cent. }}=m \omega^{2} \boldsymbol{d}$, a correction to the kinetic energy $\frac{\gamma^{2}}{2 m c}\left\{\boldsymbol{F}_{\text {Cent. }} \cdot \boldsymbol{r}, \boldsymbol{p} \cdot \boldsymbol{\alpha}\right\}$ and a correction to the mass, $\gamma^{2}\left(\boldsymbol{F}_{\text {Cent. }} \cdot \boldsymbol{r}\right)$. The third term is the spin-rotation coupling corresponding to the Coriolis force. $\boldsymbol{J}$ is the total angular momentum operator, $\boldsymbol{J}=\boldsymbol{L}+\boldsymbol{S}$.

As we are mainly concerned with molecules and solids, we continue by evaluating the nonrelativistic limit of the Dirac equation (6). We perform the Foldy-Wouthuysen transformation as described by Bjorken and Drell [41]. We rearrange the Dirac Hamiltonian as $H=\beta m c^{2}+\mathcal{O}+\mathcal{E}$, with the odd terms $\mathcal{O}=c \boldsymbol{\alpha} \cdot \boldsymbol{p}-\frac{\gamma^{2}}{2 m c}\left\{\boldsymbol{F}_{\text {Cent. }} \cdot \boldsymbol{r}, \boldsymbol{p} \cdot \boldsymbol{\alpha}\right\}$ and the even terms $\mathcal{E}=-\beta \gamma^{2}\left(\boldsymbol{F}_{\text {Cent. }} \cdot \boldsymbol{r}\right)-\boldsymbol{\omega} \cdot \boldsymbol{J}$. The transformed Hamiltonian up to the order of $\frac{1}{m c^{2}}$ is given by $H \approx \beta\left[m c^{2}+\frac{\mathcal{O}^{2}}{2 m c^{2}}+\mathcal{E}+\right.$ $\ldots$... Evaluating $\mathcal{O}^{2}$ up to $\frac{1}{m c^{2}}$ and removing the rest mass term in the particle channel gives the following Schrödinger equation, including four dynamic correction terms

$$
\begin{aligned}
\mathrm{i} \hbar \frac{\partial}{\partial t} \Psi= & {\left[\frac{\boldsymbol{p}^{2}}{2 m}-\boldsymbol{\omega} \cdot \boldsymbol{J}-\gamma^{2}\left(\boldsymbol{F}_{\text {Cent. }} \cdot \boldsymbol{r}\right)\right.} \\
& \left.-\frac{\gamma^{2}}{2 m^{2} c^{2}}\left(\boldsymbol{p}\left(\boldsymbol{F}_{\text {Cent. }} \cdot \boldsymbol{r}\right) \boldsymbol{p}+\boldsymbol{F}_{\text {Cent. }} \cdot(\boldsymbol{S} \times \boldsymbol{p})\right)\right] \Phi
\end{aligned}
$$

In comparison to Eq. (6), Eq. (7) determines a two-spinor $\Phi$ [due to the spin degrees of freedom, Eq. (7) could also be called Pauli equation].

For brevity, we omitted writing down the coupling to an electromagnetic field in this discussion. In general, we assume electrons being localized on an atom, which requires a confining potential $V(\boldsymbol{r}+\boldsymbol{d}(t))$, or, $V(\boldsymbol{r})$ in the local coordinate frame. In the nonrelativistic limit, a scalar potential in the Dirac equation leads to a scalar potential plus spin-orbit interaction and the Darwin term [41,42]. However, to lowest order, no additional corrections due to the moving frame emerge (compare also Ref. [28]). Hence writing down Eq. (7) implicitly encourages the reader to add respective potential terms.

We continue discussing the individual terms, summarized in Table I. The most prominent term is the spin-rotation coupling, or Mashoon-Zeeman term. In fact, comparing the Zeeman coupling $\mu_{B} g_{J} \boldsymbol{B} \cdot \boldsymbol{J}$ to the spin-rotation coupling allows us to identify a $1 \mathrm{THz}$ rotation with a $10 \mathrm{~T}$ magnetic field (note that the inertial spin-rotation coupling is different from the spin-rotation coupling $\sim \boldsymbol{J} \cdot \mathbf{M} \cdot \boldsymbol{I}$, coupling the electronic spin $\boldsymbol{J}$ to the nuclear spin $\boldsymbol{I}$, via the tensor $\mathbf{M}$ ). Although this seems like a significant effect, it has only been sparsely discussed in the context of rotating molecules [43]. 
TABLE I. Inertial terms arising due to an accelerated motion of the ions.

\begin{tabular}{ll}
\hline \hline Spin-rotation coupling & $\boldsymbol{\omega} \cdot \boldsymbol{J}$ \\
Centrifugal field coupling & $\gamma^{2} \boldsymbol{F}_{\text {Cent. }} \cdot \boldsymbol{r}$ \\
Centrifugal spin-orbit coupling & $\frac{\gamma^{2}}{2 m^{2} c^{2}} \boldsymbol{F}_{\text {Cent. }} \cdot(\boldsymbol{S} \times \boldsymbol{p})$ \\
Centrifugal redshift & $\frac{\gamma^{2}}{2 m^{2} c^{2}} \boldsymbol{p}\left(\boldsymbol{F}_{\text {Cent. }} \cdot \boldsymbol{r}\right) \boldsymbol{p}$ \\
\hline \hline
\end{tabular}

Furthermore, there are three terms connected to the centrifugal force. For the electron mass of $9.1 \times 10^{-31} \mathrm{~kg}$, the typical atomic distances of $1 \AA$, and vibrational frequencies of $\mathrm{THz}$, the centrifugal force is $\approx 10^{-16} \mathrm{~N}$. Comparing this value to a fictious electric field applied to an electron, this corresponds to a field strength of $\approx 10 \mathrm{Vcm}^{-1}$. In general, the action of the centrifugal force is time dependent due to the circular motion of the ions. However, the ionic motion is in the $\mathrm{THz}$ regime, corresponding to the meV energy range. Hence for sufficiently large level splitting, we apply the adiabatic approximation, assuming that the ionic motion is much slower than the electronic degrees of freedom. As a consequence, the electronic system remains in the ground state.

The first term arising due to the centrifugal force is the centrifugal field coupling $\gamma^{2} \boldsymbol{F}_{\text {Cent. }} \cdot \boldsymbol{r}$. The term occurs both in the relativistic theory (6) and in the nonrelativistic limit (7). The centrifugal field coupling is linear in the position vector $\boldsymbol{r}$ and inversion odd. Similar to the stark effect, it only introduces a correction in linear perturbation theory if inversion symmetry is broken, or if a set of degenerate levels contains parity even and odd energy levels, e.g., as for the excited hydrogen levels in the approximation of the Schrödinger equation [44]. The centrifugal field coupling requires matrix elements between states with angular momentum $l$ and $l \pm 1$, i.e., $-e\left(\Psi_{n l m} \mid \boldsymbol{E}_{p}\right.$. $\left.\boldsymbol{r} \mid \Psi_{n^{\prime} l^{\prime} m^{\prime}}\right)=\delta_{n n^{\prime}} \delta_{l, l^{\prime} \pm 1} \delta_{m m^{\prime}}\left(\Psi_{n l m}\left|\boldsymbol{E}_{p} \cdot \boldsymbol{r}\right| \Psi_{n^{\prime} l^{\prime} m^{\prime}}\right)$. For example, for the hydrogen $n=2$ states with $l=0$ and $l^{\prime}=1$, the resulting energy correction is $\Delta E= \pm 3 \gamma^{2}\left|\boldsymbol{F}_{\text {Cent. }}\right| a_{0}$, with $a_{0}$ the Bohr radius. The corresponding correction for a typical phonon is tiny, $\sim \mu \mathrm{eV}$. The same order of magnitude holds in the case of the relativistic electronic levels of the hydrogen atoms, as discussed, e.g., by Blackman and Series [45] on the example of the conventional Stark effect. Still, the level splitting due to centrifugal field is much larger than, e.g., the observable Lamb shift between the $s_{1 / 2}$ and the $p_{1 / 2}$ states of $\approx 1057 \mathrm{MHz}$ [46]. Following Marxer [47], we note that the Stark effect and the inertial field coupling roughly go as $\sim n / Z$, with $Z$ the atomic number.

The second term is the centrifugal spin-orbit coupling or Rashba-Hehl-Ni spin-orbit coupling $\sim \frac{\gamma^{2}}{2 m^{2} c^{2}} \boldsymbol{F}_{\text {Cent. }} \cdot(\boldsymbol{S} \times \boldsymbol{p})=$ $\frac{\alpha_{p}}{\hbar} \hat{\boldsymbol{z}} \cdot(\boldsymbol{\sigma} \times \boldsymbol{p})$. Note that we choose the Cartesian $z$ axis to point along the ionic displacement $\boldsymbol{d}$, i.e., in direction of the centrifugal force. The second form underlines the connection to the Rashba spin-orbit interaction. The corresponding Rashba-Hehl-Ni coefficient is given by

$$
\alpha_{p}=\frac{\hbar^{2} \omega^{2} \gamma^{2}|\boldsymbol{d}|}{4 m c^{2}}
$$

Given the electron rest mass of $\approx 0.51 \mathrm{MeV}$ and $\mathrm{THz}$ frequencies $\hbar \omega \approx 4.1 \mathrm{meV}$, the Rashba-Hehl-Ni coefficient would take values of $\alpha_{p} \approx 10^{-12} \mathrm{eVA}$. Similar to the Rashba effect, the Rashba-Hehl-Ni spin-orbit coupling is a consequence of the symmetry breaking associated to the centrifugal force. For example, starting from a spherically symmetric potential, the centrifugal force induces a symmetry breaking to a uniaxial symmetry. As a result, the Rashba-Hehl-Ni spin-orbit coupling is allowed.

The similar order of magnitude holds for the third centrifugal correction, the centrifugal redshift $\sim \frac{\gamma^{2}}{2 m^{2} c^{2}} \boldsymbol{p} \cdot\left(\boldsymbol{F}_{\text {Cent. }} \cdot \boldsymbol{r}\right) \cdot$ $\boldsymbol{p}$. It has the same prefactor as the centrifugal spin-orbit coupling, being a $\frac{1}{m c^{2}}$ correction. Similarly, as the centrifugal field coupling, the centrifugal redshift is inversion-odd, introducing a weak overlap of inversion even and odd states. In the case of periodic solids the term manifests as a correction to the dispersion relation.

To estimate the magnitude of the inertial effects, we model the ionic motion and evaluate the strength of the fictious forces. We use a generic 2D model. We excite a vibrational displacement $\boldsymbol{u}$ by an intense circularly polarized laser pulse $\boldsymbol{E}(t)$ acting in the $x y$ plane. Similarly, e.g., to Refs [7-10,48,49], the displacement is modeled by the damped classical equation of motion

$$
\ddot{\boldsymbol{d}}+\eta \dot{\boldsymbol{d}}+\mathrm{K} \boldsymbol{d}=\frac{q}{m} \boldsymbol{E}(t) .
$$

For simplicity, we couple the electric field to a localized charge, which is a good approximation for many ionic crystals. In a more realistic setup, one would couple the field to the Born-effective charge, describing the response of the macroscopic polarization per unit cell to the displacement of an atom [50]. The dynamical matrix $\mathrm{K}$ has two degenerate vibrational modes (along the Cartesian $x$ and $y$ directions) with an eigenfrequency of $f=10 \mathrm{THz}$. We choose a resonant strong circularly polarized Gaussian laser pulse with a peak field of $500 \mathrm{kVcm}^{-1}$ and a pulse width of $2 \mathrm{ps}$. The damping parameter is chosen to be $\eta=0.15 \times 2 \pi \mathrm{THz}$. We use a charge of $q=1$ and a mass of $m=10 \mathrm{u}$.

The outcome of the simulation is shown in Fig. 2. For better comparison of the strength of the centrifugal force, we introduce the pseudoelectric-field $\boldsymbol{E}_{p}$,

$$
e \boldsymbol{E}_{p}=\gamma^{2} \boldsymbol{F}_{\text {Cent. }} .
$$

Depending on the damping, the peak of the atomic displacement $(\approx 0.03 \AA)$ and the centrifugal force or pseudoelectricfield $\left(\approx 18 \mathrm{Vcm}^{-1}\right)$ emerges about $1 \mathrm{ps}$ after the peak of the initial laser field. Both the displacement and the centrifugal force decay slowly and persist for about one order of magnitude longer than the lifetime of the initial laser pulse. Hence experimentally observable consequences of inertial effects should be probed shortly after the laser pulse fully decayed.

We close the paper with a summary and an outlook. We showed that the circular motion of ions in molecules and crystals induces local inertial effects on electrons. We introduced the spin-rotation coupling corresponding to a Coriolis force and three corrections corresponding to the centrifugal force: The centrifugal field coupling; the centrifugal Rashba-HehlNi spin-orbit coupling; the centrifugal redshift. The strength of the spin-rotation coupling is significant and introduces a Zeeman-like splitting in the meV range. The strength of the centrifugal terms scale as $\sim \omega^{2} \boldsymbol{d}$. Hence they grow linearly in the displacement or rotation radius $\boldsymbol{d}$ and quadratically in 


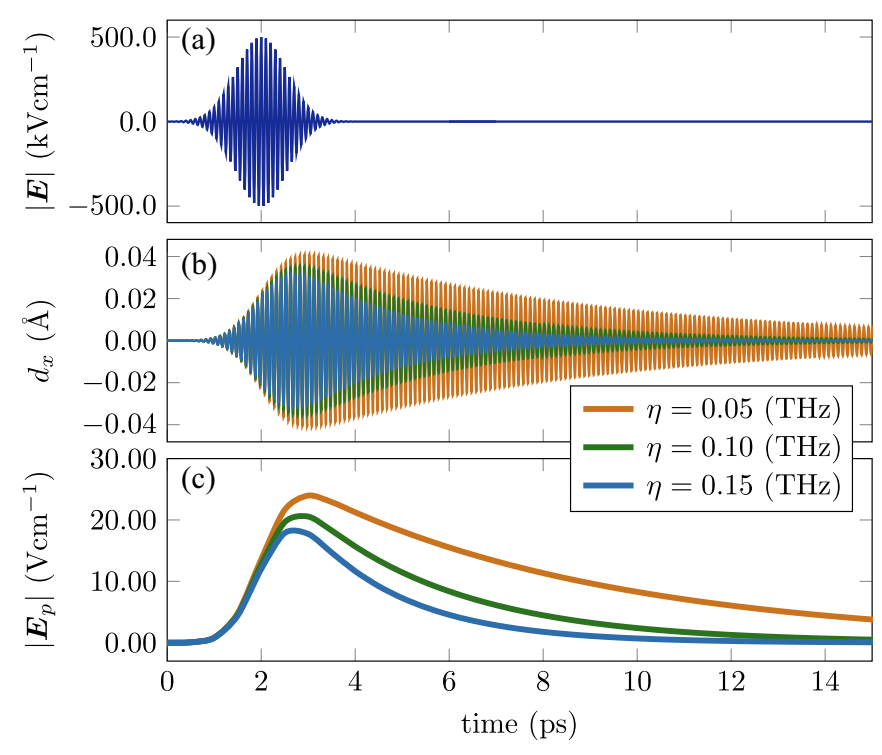

FIG. 2. (a) Initial laser pulse, (b) $x$ component of the atomic displacement, and (c) electric-field strength corresponding to the centrifugal force for a generic vibrational model. The peak of the centrifugal force arises after the peak of the initial laser pulse. Also, the lifetime of the centrifugal force is much longer than the laser pulse depending on the material-specific damping.

the rotation frequency $\omega$. Such ionic motion can be induced by circularly polarized laser pulses. We could show that the lifetime of the inertial pseudoelectric field is much longer than the lifetime of the initial laser pulse depending on the material-specific damping.

The inertial effects open new perspectives on quantum and spin control in matter. As strongly localized effects, they allow for the precise manipulation of energy levels and spin around specified ions by vibrational degrees of freedom. Vibrations and phonons can be simulated with high accuracy using stateof-the-art computational tools [51-53]. They allow to guide experiments using $\mathrm{THz}$ radiation toward a selective control of the desired quantum state. Even though inertial effects are localized on a moving ion, they will also control the overlap of electronic states in molecular or periodic systems. As a consequence, the three terms will arise in quantum manybody systems, potentially affecting quasielectron excitations in matter. Here, the effective mass of the quasielectron could be much lower than the actual electron mass, as is the case in many semiconductors. For example, in silicon the light-hole mass is about a tenth of the actual electron mass [54]. Such a lowered quasielectron mass would boost the inertial Rashba parameter $\alpha_{p} \sim m^{-1}$. In general, the challenge is to extend both $\omega$ and $\boldsymbol{d}$. Torsions in large molecules might provide the promising platform for inertial effects.

Selecting relevant target materials is outside the scope of this paper. We note that recent progress in materials informatics might allow identifying experimentally feasible materials [55-58]. The target space was defined throughout the present work. In particular, soft organic crystals, molecules, and metal organic frameworks are a promising materials class. Following implementations of static electric fields in $a b$ initio codes, we also see the prospect of implementing the inertial correction terms into $a b$ initio codes, e.g., in the framework of the density-functional perturbation theory $[59,60]$. While the majority of the discussion concerned the nonrelativistic limit, a separate discussion for the relativistic effects in matter and their implementation into ab initio codes would be necessary [42,61-63].

We are grateful to A. V. Balatsky, S. Bonetti, M. Basini, A. Brandenburg, J. D. Rinehart, V. Juričić, W. Hergert, O. Tjernberg, and M. Månson for inspiring discussions. We acknowledge support from research funding granted to A. V. Balatsky, i.e., VILLUM FONDEN via the Centre of Excellence for Dirac Materials (Grant No. 11744), the European Research Council under the European Union Seventh Framework ERS-2018-SYG 810451 HERO, the Knut and Alice Wallenberg Foundation KAW 2018.0104. Computational resources were provided by the Swedish National Infrastructure for Computing (SNIC) via the High Performance Computing Centre North (HPC2N) and the Uppsala Multidisciplinary Centre for Advanced Computational Science (UPPMAX).
[1] P. Salén, M. Basini, S. Bonetti, J. Hebling, M. Krasilnikov, A. Y. Nikitin, G. Shamuilov, Z. Tibai, V. Zhaunerchyk, and V. Goryashko, Matter manipulation with extreme terahertz light: Progress in the enabling thz technology, Phys. Rep. 836, 1 (2019).

[2] T. Kampfrath, K. Tanaka, and K. A. Nelson, Resonant and nonresonant control over matter and light by intense terahertz transients, Nat. Photon. 7, 680 (2013).

[3] M. Först, C. Manzoni, S. Kaiser, Y. Tomioka, Y.-N. Tokura, R. Merlin, and A. Cavalleri, Nonlinear phononics as an ultrafast route to lattice control, Nat. Phys. 7, 854 (2011).

[4] A. Subedi, A. Cavalleri, and A. Georges, Theory of nonlinear phononics for coherent light control of solids, Phys. Rev. B 89, 220301(R) (2014).
[5] R. Mankowsky, M. Först, and A. Cavalleri, Non-equilibrium control of complex solids by nonlinear phononics, Rep. Prog. Phys. 79, 064503 (2016).

[6] Y. T. Rebane, Faraday effect produced in the residual-ray region by the magnetic moment of an optical phonon inan ionic crystal, Sov. J. Exp. Theor. Phys. 57, 1356 (1983).

[7] D. M. Juraschek, M. Fechner, A. V. Balatsky, and N. A. Spaldin, Dynamical multiferroicity, Phys. Rev. Mater. 1, 014401 (2017).

[8] D. M. Juraschek, Q. N. Meier, M. Trassin, S. E. TrolierMcKinstry, C. L. Degen, and N. A. Spaldin, Dynamical Magnetic Field Accompanying the Motion of Ferroelectric Domain Walls, Phys. Rev. Lett. 123, 127601 (2019).

[9] R. M. Geilhufe, V. Juričić, S. Bonetti, J.-X. Zhu, and A. V. Balatsky, Dynamically induced magnetism in $\mathrm{KTaO}_{3}$, Phys. Rev. Res. 3, L022011 (2021). 
[10] M. Basini, M. Pancaldi, B. Wehinger, T. Terumasa, M. C. Hoffmann, A. V. Balatsky, and S. Bonetti, Terahertz electricfield driven magnetism in a perovskite (unpublished).

[11] B. Cheng, T. Schumann, Y. Wang, X. Zhang, D. Barbalas, S. Stemmer, and N. P. Armitage, A large effective phonon magnetic moment in a dirac semimetal, Nano Lett. 20, 5991 (2020).

[12] G. Schaack, Magnetic field dependent splitting of doubly degenerate phonon states in anhydrous cerium-trichloride, Z. Phys. B 26, 49 (1977).

[13] G. Schaack, Observation of circularly polarized phonon states in an external magnetic field, J. Phys. C 9, L297 (1976).

[14] D. M. Juraschek, T. Neuman, and P. Narang, Giant phonon-induced effective magnetic fields in $4 f$ paramagnets, arXiv:2007.10556.

[15] A. Baydin, F. G. Hernandez, M. Rodriguez-Vega, A. K. Okazaki, F. Tay, G. T. Noe II, I. Katayama, J. Takeda, H. Nojiri, P. H. Rappl et al., Magnetic control of soft chiral phonons in pbte, arXiv:2107.07616.

[16] D. M. Juraschek and N. A. Spaldin, Orbital magnetic moments of phonons, Phys. Rev. Mater. 3, 064405 (2019).

[17] L. Zhang and Q. Niu, Angular Momentum of Phonons and the Einstein-de Haas Effect, Phys. Rev. Lett. 112, 085503 (2014).

[18] D. A. Garanin and E. M. Chudnovsky, Angular momentum in spin-phonon processes, Phys. Rev. B 92, 024421 (2015).

[19] A. Rückriegel, S. Streib, G. E. W. Bauer, and R. A. Duine, Angular momentum conservation and phonon spin in magnetic insulators, Phys. Rev. B 101, 104402 (2020).

[20] Y. Ren, C. Xiao, D. Saparov, and Q. Niu, Phonon Magnetic Moment from Electronic Topological Magnetization, Phys. Rev. Lett. 127, 186403 (2021)

[21] H. Fröhlich, Theory of electrical breakdown in ionic crystals, Proc. R. Soc. Lond. A 160, 230 (1937).

[22] R. D. Mattuck and M. Strandberg, Spin-phonon interaction in paramagnetic crystals, Phys. Rev. 119, 1204 (1960).

[23] T. Shimanouchi, Tables of Molecular Vibrational Frequencies (US Government Printing Office, 1973).

[24] S. Bell, Ab initio study of the barriers to methyl torsion and torsional frequencies of acetyl molecules, Spectrochim. Acta 61, 1471 (2005).

[25] B. Yu, F. Zeng, Y. Yang, Q. Xing, A. Chechin, X. Xin, I. Zeylikovich, and R. Alfano, Torsional vibrational modes of tryptophan studied by terahertz time-domain spectroscopy, Biophys. J. 86, 1649 (2004).

[26] S. A. Werner, J.-L. Staudenmann, and R. Colella, Effect of Earth's Rotation on the Quantum Mechanical Phase of the Neutron, Phys. Rev. Lett. 42, 1103 (1979).

[27] B. Mashhoon, Neutron Interferometry in a Rotating Frame of Reference, Phys. Rev. Lett. 61, 2639 (1988).

[28] B. Müller and W. Greiner, The two centre dirac equation, Z. Naturforschung A 31, 1 (1976).

[29] F. W. Hehl and W.-T. Ni, Inertial effects of a dirac particle, Phys. Rev. D 42, 2045 (1990).

[30] L. Ryder, Spin-rotation coupling and fermi-walker transport, Gen. Relativ. Gravit. 40, 1111 (2008).

[31] A. Danner, B. Demirel, W. Kersten, H. Lemmel, R. Wagner, S. Sponar, and Y. Hasegawa, Spin-rotation coupling observed in neutron interferometry, npj Quantum Inf. 6, 23 (2020).

[32] F. W. Hehl, On the kinematics of the torsion of space-time, Found. Phys. 15, 451 (1985).
[33] B. Basu and D. Chowdhury, Inertial effect on spin-orbit coupling and spin transport, Ann. Phys. 335, 47 (2013).

[34] M. Matsuo, J. Ieda, E. Saitoh, and S. Maekawa, Spin-dependent inertial force and spin current in accelerating systems, Phys. Rev. B 84, 104410 (2011).

[35] K. Neeraj, N. Awari, S. Kovalev, D. Polley, N. Z. Hagström, S. S. P. K. Arekapudi, A. Semisalova, K. Lenz, B. Green, J.-C. Deinert et al., Inertial spin dynamics in ferromagnets, Nat. Phys. 17, 245 (2021).

[36] S. Bhattacharjee, L. Nordström, and J. Fransson, Atomistic Spin Dynamic Method with Both Damping and Moment of Inertia Effects Included from First Principles, Phys. Rev. Lett. 108, 057204 (2012).

[37] D. Böttcher, A. Ernst, and J. Henk, Atomistic magnetization dynamics in nanostructures based on first principles calculations: Application to co nanoislands on cu (111), J. Phys.: Condens. Matter 23, 296003 (2011).

[38] M.-C. Ciornei, J. M. Rubí, and J.-E. Wegrowe, Magnetization dynamics in the inertial regime: Nutation predicted at short time scales, Phys. Rev. B 83, 020410(R) (2011).

[39] F. W. Hehl, J. Lemke, and E. W. Mielke, Two lectures on fermions and gravity, in Geometry and Theoretical Physics, edited by J. Debrus and A. C. Hirshfeld (Springer, Berlin, Heidelberg, 1991), pp. 56-140.

[40] C. W. Misner, K. S. Thorne, and J. A. Wheeler, Gravitation (Macmillan, 1973).

[41] J. D. Bjorken and S. D. Drell, Relativistic Quantum Mechanics (McGraw-Hill, 1964).

[42] P. Strange, H. Ebert, J. Staunton, and B. L. Gyorffy, A relativistic spin-polarised multiple-scattering theory, with applications to the calculation of the electronic structure of condensed matter, J. Phys.: Condens. Matter 1, 2959 (1989).

[43] J.-Q. Shen and S.-L. He, Geometric phases of electrons due to spin-rotation coupling in rotating $\mathrm{C}_{60}$ molecules, Phys. Rev. B 68, 195421 (2003).

[44] F. Schwabl, Quantum Mechanics (Springer, Berlin, Heidelberg, 2007).

[45] J. Blackman and G. Series, The stark effect in hydrogen and precision measurements of the rydberg constant, J. Phys. B 6 , 1090 (1973).

[46] M. I. Eides, H. Grotch, and V. A. Shelyuto, Theory of light hydrogenlike atoms, Phys. Rep. 342, 63 (2001).

[47] H. Marxer, Off-diagonal matrix elements $\left\langle n l|r k| n l^{\prime}\right\rangle$ for hydrogen-like states: An exact correspondence relationship in terms of orthogonal polynomials and the WKB approximation, J. Phys. B: At. Mol. Opt. Phys. 28, 341 (1995).

[48] A. Cartella, T. F. Nova, M. Fechner, R. Merlin, and A. Cavalleri, Parametric amplification of optical phonons, Proc. Natl. Acad. Sci. USA 115, 12148 (2018).

[49] M. Kozina, M. Fechner, P. Marsik, T. van Driel, J. M. Glownia, C. Bernhard, M. Radovic, D. Zhu, S. Bonetti, U. Staub et al., Terahertz-driven phonon upconversion in $\mathrm{SrTiO}_{3}$, Nat. Phys. 15, 387 (2019).

[50] P. Ghosez, J.-P. Michenaud, and X. Gonze, Dynamical atomic charges: The case of $\mathrm{ABO}_{3}$ compounds, Phys. Rev. B 58, 6224 (1998).

[51] S. Baroni, S. de Gironcoli, A. Dal Corso, and P. Giannozzi, Phonons and related crystal properties from density-functional perturbation theory, Rev. Mod. Phys. 73, 515 (2001). 
[52] A. Togo and I. Tanaka, First principles phonon calculations in materials science, Scr. Mater. 108, 1 (2015).

[53] Y. Wang, S.-L. Shang, H. Fang, Z.-K. Liu, and L.-Q. Chen, First-principles calculations of lattice dynamics and thermal properties of polar solids, npj Comput. Mater. 2, 16006 (2016).

[54] L. E. Ramos, L. K. Teles, L. M. R. Scolfaro, J. L. P. Castineira, A. L. Rosa, and J. R. Leite, Structural, electronic, and effectivemass properties of silicon and zinc-blende group-III nitride semiconductor compounds, Phys. Rev. B 63, 165210 (2001).

[55] R. Ramprasad, R. Batra, G. Pilania, A. Mannodi-Kanakkithodi, and C. Kim, Machine learning in materials informatics: recent applications and prospects, npj Comput. Mater. 3, 54 (2017).

[56] R. M. Geilhufe, B. Olsthoorn, and A. V. Balatsky, Shifting computational boundaries for complex organic materials, Nat. Phys. 17, 152 (2021).

[57] M. Horton, S. Dwaraknath, and K. Persson, Promises and perils of computational materials databases, Nat. Comput. Sci. 1, 3 (2021).
[58] R. L. Greenaway and K. E. Jelfs, Integrating computational and experimental workflows for accelerated organic materials discovery, Adv. Mater. 33, 2004831 (2021).

[59] K. Refson, P. R. Tulip, and S. J. Clark, Variational densityfunctional perturbation theory for dielectrics and lattice dynamics, Phys. Rev. B 73, 155114 (2006).

[60] X. Wu, D. Vanderbilt, and D. R. Hamann, Systematic treatment of displacements, strains, and electric fields in densityfunctional perturbation theory, Phys. Rev. B 72, 035105 (2005).

[61] M. Geilhufe, S. Achilles, M. A. Köbis, M. Arnold, I. Mertig, W. Hergert, and A. Ernst, Numerical solution of the relativistic single-site scattering problem for the coulomb and the mathieu potential, J. Phys.: Condens. Matter 27, 435202 (2015).

[62] J. M. Wills and A. E. Mattsson, The Dirac equation in electronic structure calculations: Accurate evaluation of DFT predictions for actinides, Tech. Rep. (LANL, Los Alamos, NM, 2012).

[63] T. Huhne, C. Zecha, H. Ebert, P. H. Dederichs, and R. Zeller, Full-potential spin-polarized relativistic Korringa-KohnRostoker method implemented and applied to bcc Fe, fcc Co, and fcc Ni, Phys. Rev. B 58, 10236 (1998). 